\title{
A Learning-Based Approach for Fast and Robust Vessel Tracking in Long Ultrasound Sequences
}

\author{
Valeria De Luca*, Michael Tschannen, Gábor Székely, and Christine Tanner \\ Computer Vision Laboratory, ETH Zürich, 8092 Zürich, Switzerland
}

\begin{abstract}
We propose a learning-based method for robust tracking in long ultrasound sequences for image guidance applications. The framework is based on a scale-adaptive block-matching and temporal realignment driven by the image appearance learned from an initial training phase. The latter is introduced to avoid error accumulation over long sequences. The vessel tracking performance is assessed on long $2 \mathrm{D}$ ultrasound sequences of the liver of 9 volunteers under free breathing. We achieve a mean tracking accuracy of $0.96 \mathrm{~mm}$. Without learning, the error increases significantly $(2.19 \mathrm{~mm}, \mathrm{p}<0.001)$.
\end{abstract}

Keywords: tracking, block-matching, learning, real-time, ultrasound.

\section{Introduction}

During conformal radiation therapies, motion in the treatment region needs to be compensated to ensure accuracy of the dose delivery. For the thorax and abdomen, motion due to respiration is substantial and can not be neglected [1121. Image-guided radiation therapies use image information gathered during therapy for adjusting the treatment plan. Tracking the respiratory motion on such images requires an accuracy in the millimeter range and real-time feedback. Potential imaging techniques for guidance include CT, MRI and ultrasound (US). The latter represents the only modality that is real-time, non-ionizing and cheap.

Structures on US sequences of the abdomen have been tracked using optical flow [5], speckle tracking algorithms [8], intensity-based registration [22], active contours [23, hybrid methods 6 6133] and US imaging models [12]. Blockmatching algorithms (BMAs) compute the local displacements from interpolating the translations that provide the best match of image regions in two consecutive frames. Many BMAs have been proposed (e.g. [11914 28]), yet their performance has so far only been assessed on relative short sequences $(<1 \mathrm{~min})$. Therapy guidance requires the tracking of long sequences, which poses a special challenge for BM due to its iterative nature. Moreover, our sequences suffer from noise, interferences, low SNR and frame dropouts. To create a robust framework for feature tracking in long sequences, we propose an algorithm, which combines several BM components and includes a novel adaptation of the block size to

\footnotetext{
* We thank the Swiss National Science Foundation (CRSII2 127549) for funding.
} 
the feature scale. In addition, we exploit the approximate periodicity of breathing motion for learning image appearance and corresponding motion behavior (extracted by accurate but slow image registration) to allow frequent temporal realignment of the BMA for drift-free real-time tracking.

\section{Material}

US liver sequences of 9 volunteers during free breathing were acquired at the Geneva University Hospital 17. To evaluate US tracking performance for hybrid US and MR guided treatments [17, an Acuson clinical scanner (Antares; Siemens Medical Solutions, Mountain View, CA) was modified to be MR compatible, and US and MR images were simultaneously acquired. The US images (realtime second harmonic images with 1.8-2.2 MHz center frequency) were exported on-the-fly using a frame grabber device. 2D US images were acquired at a fixed location (longitudinal or intercostal plane) over 5:21, 5:28 and 10:08 min for 1, 7 and 1 volunteer(s), respectively. The resulting 2650 to 14516 frames had a temporal and spatial resolution of $14-25 \mathrm{~Hz}$ and $0.3-0.7 \mathrm{~mm}$, respectively.

\section{Method}

\subsection{Scale-adaptive Block-matching}

The key components of our proposed scale-adaptive BMA (SA-BMA) are a novel adaptation of the block size to the feature scale and the new combination of the interpolation function from [14] with the temporal realignment from [19].

Block Configuration. Traditionally the size of the blocks is chosen empirically [167] or equal to the US speckle size [10. We adapt the block size to the feature size to ensure that every block contains a part of the feature, which limits the aperture problem and avoids ambiguities due to homogeneous blocks.

The position of features to track, e.g. $P_{j}\left(t_{0}\right)$ for vessel $j$, are manually selected in $I\left(t_{0}\right)$, see Fig. 1 $\mathrm{BM}$ is performed for a region of interest $\left(R O I_{j}\left(t_{0}\right)\right)$ around feature $j$, which covers a $M \mathrm{x} N$ grid of equally sized squares (called blocks) $B_{i, j}$ of size $\Delta b_{j}$ with center points $G_{i, j}, i \in[1, \ldots, M N]$, defined at $t_{0} . \Delta b_{j}$ is determined from the feature size. In detail, as vessel cross sections have elliptic shape, we search for blob-like features centered at $P_{j}$. A scale-space approach (local maxima of a Difference-of-Gaussian (DoG)) [15ा20] is used to detect the most likely blob in $R O I_{j}\left(t_{0}\right)$. The resulting scale $s$ is related to the minor semiaxis $r_{j}$ of an ellipse fitted to the vessel section by $r_{j}=\sqrt{2 s}$ and $\Delta b_{j}=\left\lceil r_{j}\right\rceil$.

Displacement Calculation. We compute the motion field in each $R O I_{j}$ by determining the displacement at $G_{i, j}$ via BM, and use weighted interpolation [14] to obtain the displacement of $P_{j}$. At time step $t^{*}$ the displacement of $G_{i, j}\left(t^{r e f}\right)$ in the reference frame $t^{r e f}$ to $G_{i, j}\left(t^{*}\right)$, denoted as $\mathbf{d}_{G_{i, j}}\left(t^{*}\right)$, is determined by the displacement $\mathbf{v}$ which maximized the normalized cross-correlation (NCC) between $B_{i, j}\left(t^{r e f}\right)$ and the block from $I\left(t^{*}\right)$ centered at $G_{i, j}\left(t^{r e f}\right)+\mathbf{v}$. The values 
of $\mathbf{v}$ are restricted to cover only a certain search region. The reference frame is generally the previous frame $\left(t^{*}-1\right)$. Other strategies for $t^{r e f}$ are described in the next paragraph. The displacement of the tracked point from $t^{r e f}$ to $t^{*}\left(\mathbf{d}_{j}\left(t^{*}\right)\right)$ is deduced from the block displacements $\mathbf{d}_{G_{i, j}}\left(t^{*}\right)$ by weighted interpolation:

$$
\mathbf{d}_{j}\left(t^{*}\right)=\sum_{\hat{i}} w_{\hat{i}} \mathbf{d}_{G_{\hat{i}, j}}\left(t^{*}\right)
$$

where $w_{\hat{i}}$ are the weights and $\hat{i}=\left\{i \mid Q\left(i, t^{*}\right)=1\right\} . Q\left(i, t^{*}\right)$ is the filtering mask for $R O I_{j}$ at time $t^{*}$, which is defined by $Q\left(i, t^{*}\right)=1$ for the $9 G_{i, j}\left(t^{r e f}\right)$ closest to $P_{j}\left(t^{r e f}\right)$, and $Q\left(i, t^{*}\right)=0$ otherwise. We consider the weights $w_{\hat{i}}[14$ :

$$
w_{\hat{i}}=0.5 \frac{1}{D_{\hat{i}}^{2}+1} \frac{1}{\sum_{\hat{i}} \frac{1}{D_{\hat{i}}^{2}+1}}+0.5 \frac{\alpha_{\hat{i}}}{\sum_{\hat{i}} \alpha_{\hat{i}}},
$$

with $D_{\hat{i}}$ the Euclidean distance from $G_{\hat{i}, j}\left(t^{r e f}\right)$ to $P_{j}\left(t^{r e f}\right)$, and $\alpha_{\hat{i}}=\sigma_{\hat{i}}^{2} / \mu_{\hat{i}}$ the ratio between the variance $\left(\sigma_{\hat{i}}^{2}\right)$ and the mean $\left(\mu_{\hat{i}}\right)$ of the pixel intensities in $B_{\hat{i}, j}\left(t^{*}\right)$. This interpolation scheme has the advantage that it incorporates regularization (first term) and accounts for the relative image content (second term) [14. The position of the tracked point is $P_{j}\left(t^{*}\right)=P_{j}\left(t^{r e f}\right)+\mathbf{d}_{j}\left(t^{*}\right)$.

Reference Frame Definition. BMAs can generally only cope with small deformations and appearance changes, as they are based on the translations of local regions. Hence $\mathrm{BM}$ is applied to temporally consecutive frames (i.e. $\left.t^{r e f}=t^{*}-1\right)$ for tracking. However, this strategy is subject to error accumulation leading to drift. Such errors are particularly relevant in long sequences. Yet the approximate periodic nature of respiratory motion provides frequently frames which are similar to the initial frame and BM is again applicable for aligning these [19]. Errors occur also due to the quantization of $\mathbf{d}_{G_{\hat{i}, j}}$. Hence we introduce the following strategy:

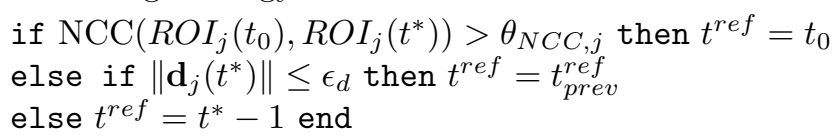

where $\operatorname{NCC}(A, B)$ is the $\mathrm{NCC}$ between image region $A$ and $B, \theta_{N C C, j}$ is the 84 th percentile of the NCC values gathered from an initial subset of the sequence, $\epsilon_{d}=0.01$ pixel, and $t_{\text {prev }}^{r e f}$ denotes $t^{r e f}$ from the previous image pair.

\subsection{Learning-based Tracking}

During therapy, images are acquired continuously over several minutes. Hence temporal realignment of the images is crucial to ensure robust tracking and to avoid error accumulation. For repetitive motion, such as breathing, redundancy within the images can be exploited [4]. Following a similar strategy, we divide the method into a training and tracking phase. During training we learn the relationship between image appearance and the corresponding displacements, from a slower, but more robust tracking method. During the clinical application, 
the displacements are computed by the proposed SA-BMA (see Sec. 3.1), with the reference frame given by the closest frame from the training set. This strategy allows temporal realignment over many more breathing states than previously.

Training Phase. In the training phase we acquire a sequence covering 10 breathing cycle, resulting in $T_{10 C}$ images $I\left(t_{i}\right), t_{i} \in\left[t_{0}, \ldots, T_{10 C}\right]$.

The images $I\left(t_{i}\right)$ are registered to $I\left(t_{0}\right)$, to obtain spatial correspondence. The registration optimizes the parameters of an affine transformation with respect to NCC over a manually selected region around $P_{j}\left(t_{0}\right)$ and is initialized by the result from $I\left(t_{i-1}\right)$ to $I\left(t_{0}\right)$.

To store the image appearance efficiently, we embed the images $I\left(t_{i}\right) \in \mathbb{R}^{D}$ into a low-dimensional representation $\mathbf{S}\left(t_{i}\right)=\left[s_{1}\left(t_{i}\right) ; \ldots ; s_{L}\left(t_{i}\right)\right] \in \mathbb{R}^{L}$, with $L \ll D$, using Principal Component Analysis (PCA) 4 . We select $L$ such that the cumulative energy of the first $L$ eigenvectors just exceeds $95 \%$. In addition, we select the PCA component $\mathbf{s}_{B}$ in $\mathbf{S}$ that captures the main breathing motion, by computing the FFT of each $\mathbf{s}_{i}$ and choosing the one that has a power spectral density maximum at $0.15-0.4 \mathrm{~Hz}(2.5-6 \mathrm{~s}$, common breathing). $\mathbf{S}$ and the corresponding registration results (e.g. $P_{j}$ ) are stored $\forall t_{i}$.

Tracking Phase. New images are continuously acquired during treatment. Given the current image $I\left(t^{*}\right)$, we first project it into the PCA space $\left(\mathbf{S}\left(t^{*}\right)=\right.$ $\left.\left[s_{1}\left(t^{*}\right) ; \ldots ; s_{L}\left(t^{*}\right)\right]\right)$. Then, depending on its similarity to the training data and the previous frame, a reference frame is chosen. The logic is as follows:

outlierFlag $=$ false

if $\left\|\mathbf{S}\left(t^{*}\right)-\mathbf{S}\left(t_{0}\right)\right\|_{2}<\theta_{1}$ then $t^{r e f}=t_{0}$

else if $\operatorname{argmin}_{t_{x} \in\left[t_{0}, \ldots, T_{10 C}\right]} \mid \mathbf{S}\left(t^{*}\right)-\mathbf{S}\left(t_{x}\right) \|_{2}<\theta_{2}$ then $t^{r e f}=t_{x}$

else if $\left\|\mathbf{S}\left(t^{*}\right)-\mathbf{S}\left(t^{*}-1\right)\right\|_{2}<\theta_{2}$ then $t^{r e f}=t^{*}-1$

else outlierFlag $=$ true end

if (outlierFlag $==$ false) then do SA-BMA

else do affine registration and update $\mathbf{S}$ end

The threshold $\theta_{1}$ is the 5 th percentile of the Euclidean distance between $\mathbf{S}\left(t_{0}\right)$ and $\mathbf{S}\left(t_{i}\right) \forall t_{0}<t_{i} \leq T_{10 C} . \theta_{2}$ is the 95th percentile of the distribution of the minimum Euclidean distances between the $\mathbf{S}\left(t_{i}\right)$ in the training set [4].

\subsection{Evaluation}

We compared the performance of SA-BMA (Sec. 3.1) and LB-BMA (Sec. 3.2). As baseline BMA, we modified the SA-BMA to have fixed block size $\Delta b_{j}=16$. The methods were tested for a total of 25 vessels in 9 sequences, see Fig. 1. We visually inspected the tracking quality for all vessels. We quantitatively evaluated the tracking error for the 15 vessels, which appeared to allow reliable annotations. We randomly selected $10 \%$ of the tracking phase images and manually annotated the position (denoted as $\bar{P}_{j}$ ) corresponding to $P_{j}\left(t_{0}\right)$. For the annotated frame $(\hat{t})$, we calculated the tracking error $\mathrm{TE}_{j}(\hat{t})=\left\|P_{j}(\hat{t})-\bar{P}(\hat{t})\right\|$. We summarize the results by the mean (MTE), standard deviation (SD) and 95th percentile of all $\mathrm{TE}(\hat{t})$, considering all landmarks as a single distribution. We computed the 


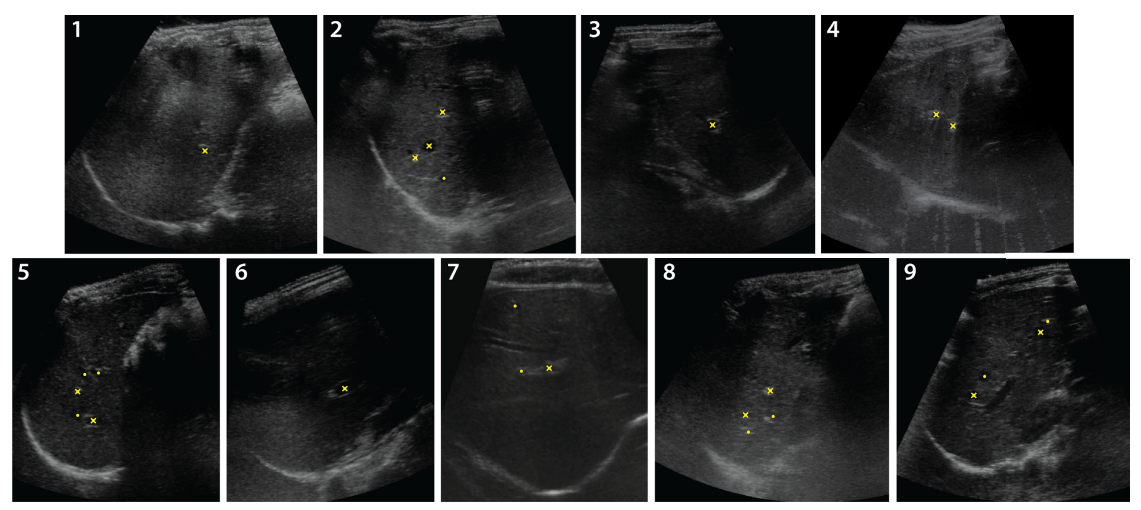

Fig. 1. $I\left(t_{0}\right)$ of the 9 sequences and manual annotation of the tracked vessel centers $P_{j}\left(t_{0}\right), j \in[1, \ldots, 25]$. Quantitative evaluation was based on the $15 P_{j}$ marked by 'x'. Visible artifacts include MR-RF interferences (4), and small acoustic windows $(2,3,6,8)$.

MTE for each landmark $j\left(\mathrm{MTE}_{j}\right)$ and report the range for the 15 vessels. We included the motion magnitude of the vessels, i.e. $\left\|P_{j}\left(t_{0}\right)-\bar{P}(\hat{t})\right\|$.

We estimated the inter-observer variability of the annotations. Two additional experts annotated $3 \%$ of randomly selected images from the tracking phase. We then defined as ground truth the mean position over the 3 annotations and calculated the tracking error as before.

\section{Results}

We tracked a total of $\sim 50000$ frames, acquired over a total of $\sim 50$ min. $\Delta b_{j}$ ranges in $[4,22]$ pixels and the size of the tracked vessels varies from 2 to $9 \mathrm{~mm}$. The PCA space is characterized by $L$ in the range of [86, 287], vs. $D>3660$.

We firstly evaluated the registration error for the training images. The affine registration achieves an accuracy of $0.63 \pm 0.36 \mathrm{~mm}(1.30 \mathrm{~mm})$ on average (MTE $\pm \mathrm{SD}$ (95th percentile of TE)), with a $\mathrm{MRE}_{j}$ range of $[0.42,0.84] \mathrm{mm}$.

Table 1 lists the results for the proposed approaches, SA-BMA and LB-BMA.

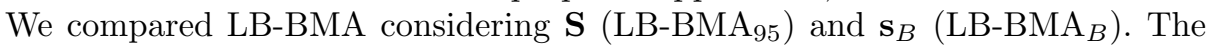
best performance is achieved by LB-BMA 95 with a MTE of $0.96 \mathrm{~mm}$. Fig. 2 2 illustrates the benefit of the proposed methods for the worst case of BMA. For LB$\mathrm{BMA}_{95}\left(\mathrm{LB}-\mathrm{BMA}_{B}\right)$ and all 25 tracked vessels, $t^{\text {ref }}$ is picked from the training set for $68.3 \%$ (99.0\%) of the frames, while 1.4\% (1.0\%) require affine registration.

The inter-observer MTE varies from 0.30 to $0.34 \mathrm{~mm}$ (95th TE from 0.63 to $0.68 \mathrm{~mm}, \mathrm{MTE}_{j}$ range $\left.[0.16,0.70]\right)$. For the inter-observer data set, the median $\mathrm{TE}_{j}$ of BMA and SA-BMA, SA-BMA and LB-BMA ${ }_{B}$, and SA-BMA and LB-BMA $_{95}$ were statistically significantly different at the 0.001 level (Wilcoxon sign-rank test), while LB-BMA ${ }_{B}$ and $\mathrm{LB}^{-B M A_{95}}$ were not $(\mathrm{p}=0.53)$. No other statistical tests were performed. 
Table 1. Tracking results (in $\mathrm{mm}$ ) for the different methods w.r.t. manual annotation from one and three observers. Best results are in bold face.

\begin{tabular}{|c|c|c|c|c|}
\hline & \multicolumn{2}{|c|}{1 Obs $-10 \%, \sim 7500$ images } & \multicolumn{2}{|c|}{3 Obs $-3 \%, \sim 2500$ images } \\
\hline & $\mathrm{MTE} \pm \mathrm{SD}\left(95^{t h} \mathrm{TE}\right)$ & rangeMTE $_{j}$ & $\mathrm{MTE} \pm \mathrm{SD}\left(95^{t h} \mathrm{TE}\right)$ & rangeMTE $_{j}$ \\
\hline VesselMotion & $5.17 \pm 3.21$ & {$[2.81,11.48]$} & $5.22 \pm 3.23(10.57)$ & {$[3.00,11.30]$} \\
\hline BMA & $3.22 \pm 2.26(7.24)$ & {$[1.25,12.35]$} & $3.20 \pm 2.26(7.17)$ & {$[1.26,12.16]$} \\
\hline SA-BMA & $2.19 \pm 1.46(4.90)$ & {$[1.20,5.79]$} & $2.18 \pm 1.45(4.83)$ & {$[1.22,5.78]$} \\
\hline $\mathrm{LB}_{-\mathrm{BMA}_{B}}$ & $1.24 \pm 1.41(3.81)$ & {$[1.04, \mathbf{1 . 4 9}]$} & $1.21 \pm 1.39(3.67)$ & {$[0.98, \mathbf{1 . 4 9}]$} \\
\hline $\mathrm{LB}_{-\mathrm{BMA}} 95$ & $0.96 \pm 0.64(2.26)$ & {$[\mathbf{0 . 3 8}, 2.34]$} & $0.97 \pm 0.65(2.20)$ & {$[\mathbf{0 . 3 6}, 2.24]$} \\
\hline
\end{tabular}

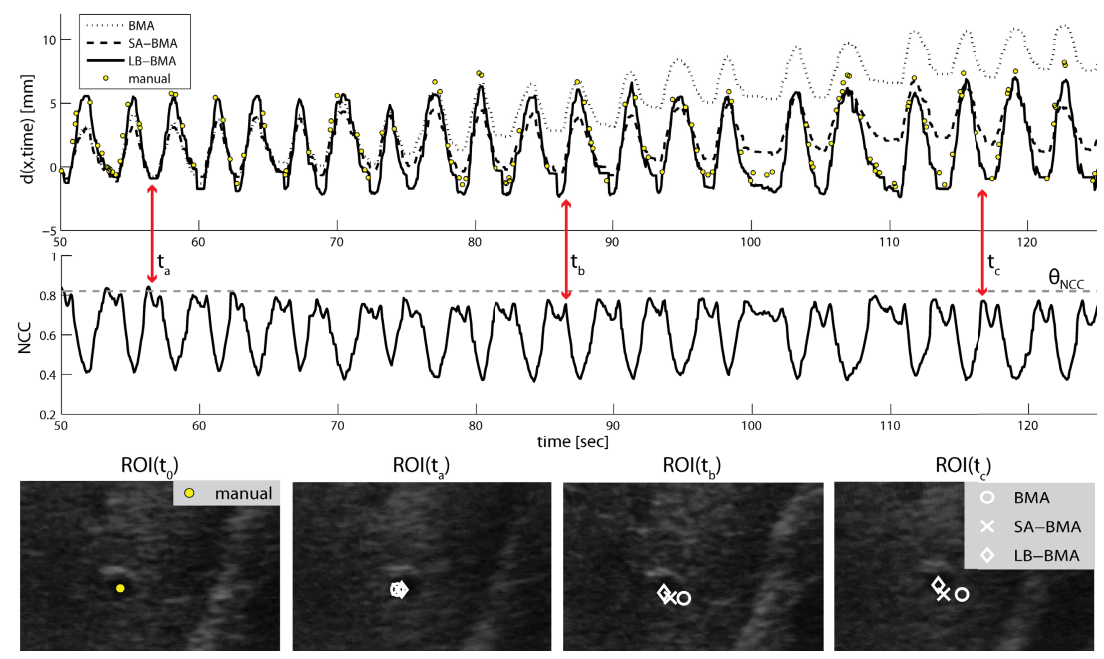

Fig. 2. Comparison of the tracking performance for a sequence where BMA failed $\left(\mathrm{MTE}_{j}=12.4 \mathrm{~mm}\right)$. (Top) Main motion component of manual annotation and $d_{j}$ from 3 methods for a temporal subset. (Middle) Corresponding NCC to first image. (Bottom, left to right) First image with annotation $\left(P\left(t_{0}\right)\right)$, image with tracking results at last realignment $\left(t_{a}\right)$ of $(\mathrm{SA}-) \mathrm{BMA}$, at $t_{a}+30 \mathrm{~s}$ and $t_{a}+60 \mathrm{~s}$. Drift occurs in a significant (moderate) way for BMA (SA-BMA) for $t>t_{a}$, while LB-BMA remains robust.

The average time to compute the motion of the tracked vessel per frame was $100 \mathrm{~ms}$ (range [30, 350] ms). The PCA projection in LB-BMA required $\sim 13 \mathrm{~ms}$ per frame. These measures were obtained using non-optimized Matlab software and no GPU parallel computing (single PC with IntelßCore ${ }^{\text {MM }_{\mathrm{i}} \text {-920 }}$ at $2.66 \mathrm{GHz}$ processor and $8 \mathrm{~GB}$ RAM), and exclude outliers, i.e. images that required affine registration. The latter was computed in approximately 0.8-2.5 s per image region, using the Insight Segmentation and Registration Toolkit (ITK). 


\section{Conclusion}

We proposed a novel and robust framework for vessel tracking in long US sequences. The method is based on learning the relationship between image appearance and feature displacements to allow frequent reinitialization of a scaleadaptive block-matching algorithm. The method was evaluated on long US sequences of the liver of 9 volunteers under free breathing and achieved a mean accuracy of $0.96 \mathrm{~mm}$ for tracking vessels for 5-10 min. To our knowledge, this is the first evaluation for tracking such long US sequences. Our performance also improves the state-of-the-art in 2D US tracking of the human liver (1.6 mm [23]). The proposed method is robust to interference, noise (see Fig. 1), and frame dropouts. Moreover, it is potentially real-time [9184].

Standard BMA might fail in long sequences, due to an inappropriate block size, changes in the image similarity values and error accumulation. The introduction of scale-adaptive blocks and the learning strategy were both significant for the improvement of the results. While adaption to the feature size reduces the error caused by ambiguous matches, the use of NCC for measuring the feasibility of temporal realignment can be misleading. Even with adaptation to the individual US sequence, temporal realignment of the tracking was often too sparse. In contrast, the proposed learning based approach enables more frequent realignments to relevant images by exploiting the repetition in the images and learning the main variation in image appearance. This allows us to detect outliers and then adapt to these previously unseen variations by affine registration, which is slow but able to handle larger displacements.

Reducing computational costs by using only the breathing signal for measuring image similarity increased mean errors slightly (0.96 vs. $1.24 \mathrm{~mm})$. While affine registration performed well on the training set, it was only applied to outliers (1\%) during real-time tracking due to its computational complexity [4].

The achieved accuracy and robustness of the proposed tracking method for long and very difficult US sequences makes us confident of its success for realtime US guidance during radiation therapy under free-breathing.

\section{References}

1. Boukerroui, D., Noble, J.A., Brady, J.M.: Velocity Estimation in Ultrasound Images: A Block Matching Approach. In: Taylor, C.J., Noble, J.A. (eds.) IPMI 2003. LNCS, vol. 2732, pp. 586-598. Springer, Heidelberg (2003)

2. Byram, B., Holley, G., Giannantonio, D., Trahey, G.: 3-D phantom and in vivo cardiac speckle tracking using a matrix array and raw echo data. IEEE Trans. Ultrason. Ferroelectr. Freq. Control 57(4), 839 (2010)

3. Cifor, A., Risser, L., Chung, D., Anderson, E.M., Schnabel, J.A.: Hybrid featurebased Log-Demons registration for tumour tracking in 2-D liver ultrasound images. In: Proc. IEEE Int. Symp. Biomed. Imaging, p. 724 (2012)

4. De Luca, V., Tanner, C., Szekely, G.: Speeding-up Image Registration for Repetitive Motion Scenarios. In: Proc. IEEE Int. Symp. Biomed. Imaging, p. 1355 (2012)

5. Demi, M., Bianchini, E., Faita, F., Gemignani, V.: Contour tracking on ultrasound sequences of vascular images. Pattern Recognition and Image Anal. 18, 606 (2008) 
6. Foroughi, P., Abolmaesumi, P., Hashtrudi-Zaad, K.: Intra-subject elastic registration of 3D ultrasound images. Med. Image Anal. 10(5), 713 (2006)

7. Harris, E.J., Miller, N.R., Bamber, J.C., Evans, P.M., Symonds-Tayler, J.R.N.: Performance of ultrasound based measurement of 3D displacement using a curvilinear probe for organ motion tracking. Phys. Med. Biol. 52(18), 5683 (2007)

8. Harris, E.J., Miller, N.R., Bamber, J.C., Symonds-Tayler, J.R.N., Evans, P.M.: Speckle tracking in a phantom and feature-based tracking in liver in the presence of respiratory motion using 4D ultrasound. Phys. Med. Biol. 55(12), 3363 (2010)

9. Hsu, A., Miller, N.R., Evans, P.M., Bamber, J.C., Webb, S.: Feasibility of using ultrasound for real-time tracking during radiotherapy. Med. Phys. 32(6), 1500 (2005)

10. Kaluzynski, K., Chen, X., Emelianov, S.Y., Skovoroda, A.R., O'Donnell, M.: Strain rate imaging using two-dimensional speckle tracking. IEEE Trans. Ultrason. Ferroelectr. Freq. Control 48(4), 1111 (2001)

11. Keall, P.J., Mageras, G.S., Balter, J.M., Emery, R.S., Forster, K.M., Jiang, S.B., Kapatoes, J.M., Low, D.A., Murphy, M.J., Murray, B.R., Ramsey, C.R., Van Herk, M.B., Vedam, S.S., Wong, J.W., Yorke, E.: The management of respiratory motion in radiation oncology report of AAPM Task Group 76. Med. Phys. 33, 3874 (2006)

12. King, A.P., Rhode, K.S., Ma, Y., Yao, C., Jansen, C., Razavi, R., Penney, G.P.: Registering preprocedure volumetric images with intraprocedure 3-D ultrasound using an ultrasound imaging model. IEEE Trans. Med. Imaging 29(3), 924 (2010)

13. Leung, C., Hashtrudi-Zaad, K., Foroughi, P., Abolmaesumi, P.: A Real-Time Intrasubject Elastic Registration Algorithm for Dynamic 2-D Ultrasound Images. Ultrasound. Med. Biol. 35(7), 1159 (2009)

14. Lin, C.H., Lin, M.C.J., Sun, Y.N.: Ultrasound motion estimation using a hierarchical feature weighting algorithm. Comput. Med. Imaging Graph. 31(3), 178 (2007)

15. Lindeberg, T.: Feature detection with automatic scale selection. Int. J. Comput. Vision 30, 79 (1998)

16. Morsy, A.A., Von Ramm, O.T.: FLASH correlation: a new method for 3-D ultrasound tissue motion tracking and blood velocity estimation. IEEE Trans. Ultrason. Ferroelectr. Freq. Control 46(3), 728 (1999)

17. Petrusca, L., Cattin, P., De Luca, V., Preiswerk, F., Celicanin, Z., Auboiroux, V., Viallon, M., Arnold, P., Santini, F., Terraz, S., Scheffler, K., Becker, C.D., Salomir, R.: Hybrid Ultrasound/Magnetic Resonance Simultaneous Acquisition and Image Fusion for Motion Monitoring in the Upper Abdomen. Invest. Radiol. 48, 333 (2013)

18. Pinton, G.F., Dahl, J.J., Trahey, G.E.: Rapid tracking of small displacements with ultrasound. IEEE Trans. Ultrason. Ferroelectr. Freq. Control 53(6), 1103 (2006)

19. Revell, J., Mirmehdi, M., McNally, D.: Computer Vision Elastography: Speckle Adaptive Motion Estimation for Elastography Using Ultrasound Sequences. IEEE Trans. Med. Imaging 24(6), 755 (2005)

20. Schneider, R.J., Perrin, D.P., Vasilyev, N.V., Marx, G.R., del Nido, P.J., Howe, R.D.: Real-time image-based rigid registration of three-dimensional ultrasound. Med. Image Anal. 16, 402 (2012)

21. Shirato, H., Shimizu, S., Kitamura, K., Onimaru, R.: Organ motion in imageguided radiotherapy: lessons from real-time tumor-tracking radiotherapy. Int. J. Clin. Oncol. 12, 8 (2007)

22. Wein, W., Cheng, J.Z., Khamene, A.: Ultrasound based Respiratory Motion Compensation in the Abdomen. In: MICCAI Workshop on Image Guidance and Computer Assistance for Soft-Tissue Interventions (2008)

23. Zhang, X., Günther, M., Bongers, A.: Real-Time Organ Tracking in Ultrasound Imaging Using Active Contours and Conditional Density Propagation. In: Liao, H., "Eddie" Edwards, P.J., Pan, X., Fan, Y., Yang, G.-Z. (eds.) MIAR 2010. LNCS, vol. 6326, pp. 286-294. Springer, Heidelberg (2010) 